\title{
Patient care: what is it?
}

\section{Diane Yorke*}

University of North Carolina, Assistant Professor in Adjunct Clinical, School of Nursing, Chapel Hill, USA

When first asked to write about patient care it seemed an easy enough task. As a healthcare provider I give and do patient care. However, I started thinking just what is it I mean by 'patient care' and what's involved when I am doing it. The http://medical-dictionary. thefreedictionary.com/Patient+care provides the following definition for care: "the services rendered by members of the health professions for the benefit of a patient" and then recommends we also see the definition of 'treatment': "care of a patient." This is too vague for me to get a real picture of what patient care means. It also equates care with treatment.

Next, I started conversations with health care professionals that I work with asking them what patient care means to them and what they do when they're providing patient care? Their answers differed a bit but all of them gave me some insight into what patient care looks like to those who provide it.

Newer health care professionals, those I arbitrarily identified as having less than 3 years of practice experience, reported that patient care is mostly doing what patients can't do for themselves. In this they included providing physical care of patients' bodies and bodily functions. In the intensive care unit setting, younger professionals described monitoring and manipulating the technology and medicines that support breathing and circulation as the patient care they do. More experienced health care professionals mentioned other aspects that in total make up their patient care: including the patient in discussions of plans and goals; providing patient education and maintaining their own professional knowledge through continuing education; addressing patients' spiritual needs; and, being supportive to patients and their families.

I was especially intrigued with the conversation I had with one of my colleagues. He describes patient care as "any interaction between a healthcare professional and healthcare consumer that is focused on healing, curing, or comfort restoration of the consumer." The example he gave was pre-op meetings with patients to plan for surgeries and how he assured patients of some beneficial result as well as assuring and providing adequate physical pain control throughout. He didn't mention families or other colleagues as being a part of his providing patient care but I've watched him include whomever a patient brought to his meeting and I've heard him consult with other specialists.

Other colleagues described patient care as "all encompassing," and "it's everything we do," Many of these folks had the same problem I have in truly describing patient care. Often they had difficulty even providing an example of their patient care. Finding an answer to what patient care is seemed to be getting more elusive, so I went back to the literature; always go back the literature when you've got a question.

In nursing literature 'patient care' seems always considered in relation to something else such as a symptom, or healthcare safety, quality, and coordination. Sometimes patient care is discussed in relation to enhancing patients' quality of life. Often though, patient care is referred to with a specific patient population in mind; children and others with cancer, geriatric patients, intensive care patients, etc. A query for 'patient care' in the CINAHL data base didn't retrieve any article describing or defining 'patient care' as a separate entity.

The American Association of Critical-Care Nurses Delegation
Handbook suggests that there are two types of patient care, direct and indirect. Direct patient care refers to activities that "assist the patient in meeting their basic needs." Indirect patient care refers to activities that "focus on maintaining the environment in which nursing care is delivered and only incidentally involves direct patient care."

Pharmacists have been advised to look at patient care as a process involving 5 steps: collecting information from and about the patient, assessing this information, planning a treatment based on evidence, implementing that plan, and then following up with what happened, by monitoring and evaluating the patient's response to treatment $[1,2]$. A lack of or a negative response suggests the process begin again. The pharmacist patient care process closely resembles what I was taught in my early nursing education as the nursing care process.

Eckel $[1,2]$ goes on to state that evidenced-based practice and other health care provider perspectives are essential to the patient care process and that there are three key points all health care providers must consider in providing patient care. First, patient care must be patient centered and thus involve the patient. Patient centered care, I would add, is also family centered and include the patients' family members, however the patient defines or describes family. Second, patient care is collaborative involving coordinated communication with others involved in providing patients' health care in order that all of us work towards meeting the patients' goal. And, third, patient care requires succinct documentation of plans of care with rationale to facilitate that all care for the patient is directed towards the same patient goals. In essence then, how health care providers deliver patient care is dependent on finding out what patients want.

Patient care can be challenging. Merely defining patient care is challenging. I suggest that it is such an elusive concept because patient care looks and feels genuine and individualized. It includes, but is not limited to, anticipating and providing for each patient's needs and helping each patient achieve their health goals in light of their health condition.

\section{References}

1. Eckel F M (2015) Pharmacists' patient care process. Pharmacy Times 81.

2. Goodwin C (2016) Person-centered care: A definition and essential elements. Journal of the American Geriatrics Society 64: 15-18.

*Corresponding author: Diane Yorke, Adjunct Clinical Assistant Professor School of Nursing, University of North Carolina, Chapel Hill, USA, E-mail: dyorke@email.unc.edu

Received: April 25, 2016; Accepted: April 26, 2016; Published: May 03, 2016

Citation: Yorke D (2016) Patient care: what is it? J Pat Care 2: e101. doi: 10.4172/2573-4598.1000e101

Copyright: (c) 2016 Yorke D. This is an open-access article distributed under the terms of the Creative Commons Attribution License, which permits unrestricted use, distribution, and reproduction in any medium, provided the original author and source are credited. 